REVIEW ARTICLE

\title{
ADVANTAGES AND DISADVANTAGES OF TRANSABDOMINAL PREPERITONEAL APPROACH AND TOTAL EXTRAPERITONEAL APPROACH VERSUS OPEN REPAIR OF INGUINAL HERNIA
}

\author{
Radek Pohnan ${ }^{1,2 \varpi}$, Faye Rozwadowski ${ }^{3}$, Leo Klein ${ }^{2}$, Miroslav Ryska ${ }^{1}$ \\ ${ }^{1}$ Department of Surgery, Central Military Hospital - Faculty Military Hospital, 2nd Faculty of Medicine, Charles \\ University, Prague, Czech Republic \\ ${ }^{2}$ Department of Field Surgery, Faculty of Military Health Sciences, University of Defence, Hradec Králové, Czech \\ Republic \\ ${ }^{3}$ Naval Health Clinic Lakehurst, Lakehurst, New Jersey, U.S.A.
}

Received $18^{\text {th }}$ November 2013.

Revised $3^{\text {rd }}$ February 2013.

Published $8^{\text {th }}$ March 2013.

\begin{abstract}
Summary
Surgical repair of inguinal hernias is one of the most common surgical procedures performed. Since endoscopic inguinal hernia repair was first reported in 1990, the operation has been refined into an attractive alternative to open hernia repair for many patients and surgeons. Transabdominal preperitoneal approach (TAPP) and total extraperitoneal approach (TEP) are the most commonly used methods for miniinvasive inguinal hernia treatment. Employing the electronic databases MEDLINE, Pubmed and Ebscohost, these methods were compared with open techniques. Perioperative and postoperative complications, recurrent hernias and quality of life were evaluated. For patients with bilateral inguinal hernia or with recurrent inguinal hernia, endoscopic repair offers significant advantages over open techniques with regard to pain, recurrence risk and recovery. For unilateral primary hernias, either endoscopic or open tension - free repair with mesh can offer excellent and equivalent results.
\end{abstract}

Key words: inguinal hernia; surgery; laparoscopy; TAPP; TEP

\section{INTRODUCTION}

Inguinal hernia is the most common form of abdominal wall hernias and inguinal hernia operations are among the most frequent surgeries.

\footnotetext{
2 Central Military Hospital - Faculty Military Hospital Prague, U Vojenské nemocnice 1200, 16900 Praha 6, Czech republic

ㅁodek.pohnan@uvn.cz

ㅇㅛㅛ +420973202901
}

With the development of surgery in the second half of the $19^{\text {th }}$ century, operative repair became the essential treatment of external hernias. During the $20^{\text {th }}$ century, a large number of different operating procedures and methods were introduced for the treatment of inguinal hernia repair. Expansion of laparoscopy in abdominal surgery since the end of the last century has resulted in minimally invasive techniques [1,2].

Surgical treatment of inguinal hernia repair techniques include: traditional open technique (tension on), open technique with implanted aloplastic mate- 
rial - tension -free (Lichtenstein), and laparoscopic technique using mesh. First who described the tension - free technique with mesh was Lichtenstein in 1986. Principle of Lichtenstein technique is to cover hernia defect by plastic mesh without involving wound vascularisation by tension. Lichtenstein technique and the other types of tension - free techniques with different types of meshes practically replaced open tension - on techniques in western countries.

The number of hernia relapses is utilized to evaluate the advantages of each method. In the last decade, there has been an increase in measuring the quality of life; and last but not least the cost of individual types of procedures has been discussed [3-7].

\section{PROCEDURES}

From the laparoscopic techniques currently used worldwide, the two most used methods are: TAPP transabdominal preperitoneal approach and TEP total extraperitoneal approach. Employing the electronic databases MEDLINE, PubMed and EBSCOhost, TAPP and TEP methods were compared with the traditional open techniques. Authors identified and screened studies and reviews published in English from January 2005 to August 2012. The medical search headings (MeSH) 'inguinal hernia', 'TAPP', 'TEP', 'Lichtenstein technique' and combinations of these were used. Inclusion criteria required the studies and reviews to: (i) be written in English; (ii) be published in peer reviewed journals; (iii) provide clear documentation of the operative techniques as 'open' or 'laparoscopic'.

Intraoperative complications (injury of organs and vascular structures), postoperative complications (secondary hemorrhage, repeat operation, surgical site infection, urinary retention, wound hematoma, neuralgic pain, repeat operation due to pain, postoperative ileus, ischemic orchitis, mesh rejection, postoperative lethality, chronic pain, hernia recurrence), and quality of life were evaluated in connection with the performed type of surgery.

\section{1a. Description of laparoscopic procedures:}

For both types of procedures the same patient position is used with the patient lying on his back, upper limbs and lower limbs are adducted. The operating table is placed into a slight Trendelenburg position, the surgeon stands opposite to side of hernia and an assistant operates the laparoscope position opposite the surgeon.

\section{Transperitoneal laparoscopic approach (TAPP, transabdominal preperitoneal approach)}

Under general anesthesia, the procedure begins with the supraumbilical incision and insufflation of the abdomen. The laparoscope is introduced via the initial incision followed by the trocars as the working instruments. The most commonly used two trocars are introduced laterally via the straight abdominal muscle at the level of the umbilicus. Initially, the basic anatomical structures are identified for orientation -inferior epigastric vessels, medial umbilical ligament, ductus deferens, and spermatic vessels. In most cases the hernia is easily visualized and localized with a combined approach of outside pressure and instrumental retraction which can pull out contained visceras. The peritoneum is divided from the medial umbilical ligament to the anterior superior iliac spine. Hernia is gently dissected from the spermatic cord. After exposure of the Coopers ligament, the abdominal defect is covered by mesh. Recommended mesh size is $10 \times 15 \mathrm{~cm}[3,5-$ 9]. The most commonly used fixation points are Coopers ligament, the area of the upper medial corner and the lateral mesh edge area. For the mesh fixation, non-absorbable or absorbable tacks or clips are most frequently used. After suturing of the peritoneum over the mesh, the surgery concludes with the termination of insufflation and surgical closure.

\section{Minimally invasive preperitoneal approach (TEP, total extraperitoneal approach)}

The surgery begins under general anesthesia and an incision below the navel is performed. After incision of the upper fascia of the rectus abdominis muscle, the muscle is rectracted and with blunt dissection the preperitoneal space ipsilateral to the symphysis and the anterior superior iliac spine is prepared. Tissue expansion using a specialized catheter with a balloon is convenient, but for most surgical departments is not economically viable. Incision below the navel is used for videoport and insufflation with carbon dioxide. The first trocar is placed at the symphysis and the second working trocar is introduced at half the distance between the symphysis and the umbilicus. For anatomic orientation the following are used: pubic arch, inferior epigastric vessels, Retzs space, ductus deferens and vasa spermatica. After preparation of the hernia sac from the spermatic cord, the abdominal defect is covered by mesh. The recommended size of the mesh is $15 \times 15 \mathrm{~cm}$ [6-15]. Fixa- 
tion of the mesh is identical to the TAPP method [914]. Surgery is concluded with desufflation of carbon dioxide and surgical closure.

\section{1b. Description of open procedures:}

There have been many different open approaches developed in last two centuries. The most common method of treatment of inguinal hernia in Western countries is Lichtenstein method but many surgeons still operate using Bassini method and its variations. For both types of procedures the same patient position is used with the patient lying on his back and for both methods the same approach is used. A skin incision, extending from just below and medial to the anterosuperior iliac spine to the pubic spine, is made 2 to $3 \mathrm{~cm}$ above and parallel to Poupart's ligament. Incision is carried down to the external oblique fascia and the external ring is visualized. After incision of the external oblique fascia, spermatic cord is mobilised $[1,2,28]$.

\section{Bassini operation}

Bassini promoted repair, which included dissection of the spermatic cord, dissection of the hernia sac with high ligation, and extensive reconstruction of the floor of the inguinal canal. Following division of the cremasteric muscle and ligation of the hernia sac deep to the internal inguinal ring, a triple-layer repair is performed to restore integrity to the floor. The medial tissues, including the internal oblique muscle, transversus abdominis muscle, and transversalis fascia, are fixed to the shelving edge of the inguinal ligament and pubic periosteum with interrupted sutures. The lateral border of the repair is the medial border of the internal inguinal ring, which is subsequently reinforced by the repair [28].

\section{Lichtenstein method}

Initial exposure and mobilization of cord structures is identical to Bassini approach. The floor and internal ring are reinforced through the application of the mesh. The mesh is rectangular in shape, with a rounded edge at its apex, corresponding to the medial edge. At the other end, the mesh will be split to accommodate the spermatic cord. The rounded edge is attached to the anterior rectus sheath just medial to the pubic tubercle. The suture is then continued in a running fashion to secure the mesh around the pubic tubercle. The inferior margin of the mesh is sutured to the shelving edge of the in- guinal ligament, as the repair is continued laterally. The mesh is then tailored to fit around the cord at the internal ring. The superior and inferior flaps of the prosthesis are then placed around the base of the cord and sutured together with a single interrupted stitch. The superior edge of the mesh is then fixed to the posterior aspect of the internal oblique aponeurosis and rectus sheath, using either interrupted or continuous sutures. Nonabsorbable or long-term absorbable sutures are generally used with mesh repairs [29].

\section{Quality of life:}

Most authors assume that performing a surgery in patients with inguinal hernia will achieve improved quality of life. In many cases, however, this may not be true. If there is a serious postoperative complication, or recurrence of hernia, or in case of chronic pain in the groin, it may even significantly impair quality of life [16].

\section{RESULTS}

In comparative analyses, the number of recurrences after conventional open surgery for primary inguinal hernia ranged from $1,1 \%$ to $33 \%$ depending on the approach [3-7]. A comparison of different classical tension - on techniques shows Shouldice technique to have the best results $(1,1 \%$ incidence of recurrence in case of primary hernias) [20-22]. Other types of tension - on techniques are associated with a higher incidence of recurrence and a higher incidence of wound infections [3-6]

Surgical techniques utilizing mesh have fewer recurrences than techniques without mesh. The incidence of recurrence in tension - free Lichtenstein technique ranges from $0,7 \%$ to $2,65 \%$ for primary hernias $[3-7,23,24]$. The incidence of recurrence of endoscopic methods of TAPP and TEP is reported from 0,4 to $2,5 \%$ for primary hernias [3-9,10-19,23].

Endoscopic methods have a lower incidence of wound infections and hematomas, allowing earlier recovery and return to work, compared with Lichtenstein technique. TAPP and TEP have longer operating time and higher incidence of seromas, compared with Lichtenstein technique [3-19,23].

There is no difference between TAPP and TEP in operation time, in the incidence of hematoma, infection, vascular injury, hospitalization time, length 
and recovery time to return to normal activities and work. The learning curve with TAPP is significantly shorter $[9,13,23]$.

From the perspective of the health facility in the treatment of primary unilateral hernias, the most efficient option is an open surgery with mesh without tension due to the price to perform- ance ratio. From the socio-economic perspective, the least costly approach for working patients is endoscopic surgery, especially for those with bilateral hernias $[17,23]$.

The percentage of each type of surgery used in selected European countries is shown in Table 1.

Table 1. Representation of the different surgical techniques in selected European countries

\begin{tabular}{lccccc}
\hline \multirow{2}{*}{ Country } & Year & \multicolumn{2}{c}{ Type of inguinal hernia repair } \\
\cline { 3 - 6 } & & Conventional (\%) & Open with mesh (\%) & Endoscopically (\%) & Other method (\%) \\
\hline Netherlands & 2006 & 4 & 77 & 19 & 15 \\
\hline Denmark & 2006 & 2.5 & 82.5 & 8 & 3 \\
\hline Finland & 2006 & 7 & 46 & 34 & \\
\hline France & 2006 & 14.9 & 60 & 24 & 6 \\
\hline Poland & 2006 & 38 & & 6 & \\
\hline Austria & 2006 & 76 & 34 & 9 & \\
\hline Hungary & 2007 & 60 & 82 & & \\
\hline Sweden & 2006 & 8.5 & &
\end{tabular}

Provided by the Working Group European Hernia Society (EEC) [23].

When evaluating the quality of life after inguinal hernia surgery, endoscopic treatment achieved better results compared with open techniques using mesh $[16,17,18]$. Assessment of quality of life (QoL) in patients after inguinal hernia surgery methods TAPP, TEP and open techniques have been recently the subject of 4 trials, the results are described in Table 2.

Table 2. Studies analyzing QoL, laparoscopic and open mesh techniques (Lichteinstein method-LO)

\begin{tabular}{lccc}
\hline & Evaluated surgery & QoL questionnaire used & Result by QoL \\
\hline McCormac K et al. 2005 [17] & TAPP\&TEP vs LO & SF-36 & Laparoscopy superior \\
\hline De Jonge P et al. $2008[18]$ & TAPP\&TEP vs LO & SF-36 & Laparoscopy superior \\
\hline Srsen D et al. $\quad 2008[19]$ & TAPP vs LO & SF-36 & No difference \\
\hline
\end{tabular}

Surgical treatment is the only curative method for inguinal hernia. In 2004 in the U.S. 4787000 outpatient visits were documented and 1172 deaths were related to an abdominal wall hernia. Total costs of treating abdominal wall hernias exceeded $\$ 6$ billion. The inguinal hernia contributed 80 percent of these cases [26,27].

Efforts to treat inguinal hernias are known since ancient times. By the late $19^{\text {th }}$ century the majority of conservative treatment and surgical treatment procedures were performed rarely and only in cases of strangulation or incarceration [1,2]. Since its publication in 1889, the global use of the Bassini method was employed and many surgeons still operate using this method [28].

In 1986, Lichtenstein published his concept of repair of the inguinal hernia without tension using tissue implanted mesh [29]. This technique currently represents the most common method of treatment of inguinal hernia in Western countries [39,10-19,23].

The first laparoscopic inguinal hernia was described in 1982 by Gere [30]. In 1992, Dion and Arregui Morin developed the method of TAPP. It was 
the previous year, when Dulucq recommended total extraperitoneal access to prevent intra-abdominal injury $[1,2]$. Since the nineties there was a large expansion of this minimally invasive surgery [28], but reached no consensus on such an extension as in the case of laparoscopic cholecystectomy.

The method of choice in the treatment of primary inguinal hernias is a tension - free technique [23.31]. They can be carried out openly or endoscopically and achieve the same results [9,17-19,23,31]. Conventional tension - on operations are not recommended due to reasons of a higher incidence of recurrence and a higher incidence of wound infections [3-6]. The only exception is the Shouldice technique with a remarkably low incidence of recurrence, but this method in not commonly utilized [20-23]. In summary, in patients with primary unilateral inguinal hernia, open and endoscopic surgeries with mesh have comparable results; the choice of method depends on surgeon experience and patient preference [9.23].

Results of treatment of recurrent inguinal hernia were evaluated in several large studies. The largest was Bisgaard's study from the Danish registry of hernias [6] re-evaluation of recurrence after surgery of recurrent hernias with Lichtenstein method. Number of re-recurrence rate was $11,3 \%$ in the second time of Lichtenstein operations and 1,3\% for laparoscopic surgery.

Further prospective randomized studies evaluate the endoscopic approach as advantageous in the treatment of recurrent hernias [32-34].

The advantages of endoscopic techniques in the treatment of recurrent inguinal hernias include lower risk of orchitis and testicular atrophy and lower incidence of chronic pain, shorter recovery time, and in case of uncertainty in the diagnosis of recurrent inguinal hernia. Laparoscopy provides definitive diagnosis and additional options [35].

The main advantage of the described endoscopic procedures is for the patients with bilateral inguinal hernia, which allows the performance of minimally invasive surgery without additional incisions. Recovery time is the same as in unilateral endoscopic surgery [36]. In the Feliu prospective randomized study [37], TEP and Lichtenstein procedures were compared with 3-year follow-up. In the group treated with bilateral Lichtenstein technique, the number of complications was reported three times higher (16\% vs $5 \%$ ) and the average length of hospital stay was doubled $(1,3$ compared to 0,6 days). The number of recurrences was the same.
Some surgeons prefer the laparoscopic performance in women with inguinal hernia, due to the additional occurrence of femoral hernia, which is often overlooked and can occur up to $40 \%$ of cases [9].

With regards to the endoscopic treatment of professional athletes, the performance of endoscopic surgery does not involve the aponeurosis of the external oblique muscle and hereby minimizes scar tissue between the muscles [9].

In several randomized trials there was no difference between fixed and non-fixed mesh. According to the authors, it is not necessary to fix or adhere the mesh [10-13].

Until recently, the main factor used in assessing the success of the surgical techniques in the treatment of inguinal hernia repair was the recurrence rate.

The current focus is on chronic pain after inguinal hernia surgery repair, with more literature dealing with the type, frequency of occurrence, and risk factors [3-9,11-19,31-37].

Quality of life after inguinal hernia surgery has been observed since 1995. Although most authors would automatically believe that inguinal hernia surgical patients have improved quality of life after surgical intervention, it is shown that this may not be in the case of complications. Major intraoperative or postoperative complications, hernia recurrence, and in particular chronic pain in the groin may all decrease quality of life [16]. In studies comparing quality of life after endoscopic surgery and open inguinal hernia, better results are seen with endoscopic methods $[16,17,18]$.

In our surgical department, we treat inguinal hernias using open surgery methods - Lichtenstein technique, in selected cases we use Bassini and McVaye technique and laparoscopic TAPP technique. In the treatment of primary unilateral hernias we prefer open procedures; specifically recurrent hernia is solved laparoscopically or openly by using Lichtenstein method and double-sided hernias are mainly dealt by laparoscopic procedures. The choice of method depends on the patient's preference and surgeon's experience.

\section{CONCLUSION}

For patients with bilateral inguinal hernia or inguinal hernia recurrence, endoscopic performance is preferred over the open method with mesh due to significant advantages in terms of risk of recurrence of hernia, postoperative pain and recovery. In patients 
with primary unilateral inguinal hernia, endoscopic and open methods with mesh achieve the same results. The disadvantages of minimally invasive endoscopic procedures are the high costs and relatively long learning curve.

\section{REFERENCES:}

1. Read, RC. Crucial steps in the evolution of the preperitoneal approachesto the groin: an historical review. Hernia. 2011, 15, 1-5.

2. Read, RC. Herniology: past, present, and future. Hernia. 2009, 13, 577-580.

3. Bittner, R.; Sauerland, S.; Schmedt, CG. Comparison of endoscopic techniques vs Shouldice and other open non mesh techniques for inguinal hernia repair: a meta-analysis of randomized controlled trials. Surg Endosc. 2005, 19, 605-615.

4. Aufenacker, TJ.; de Lange, DH.; Burg, MD.; et al.. Hernia surgery changes in the Amsterdam region 1994-2001: decrease in operations for recurrent hernia. Hernia. 2005, 9, 46-50.

5. Bay-Nielsen, M.; Kehlet, H.; Strand, L.; et al., Danish Hernia Database. Quality assessment of 26, 304 herniorrhaphies in Denmark: a prospective nationwide study. Lance. 2001, 358, 1124-1128.

6. Bisgaard, T.; Bay-Nielsen, M.; Kehlet H. Rerecurrence after operation for recurrent inguinal hernia: a nationwide 8-year follow-up study on the role of type of repair. Ann Surg. 2008, 247, 707-711.

7.Shah, NR.; Mikami, DJ.; Cook, C.; et al. A comparison of outcomes between open and laparoscopic surgical repair of recurrent inguinal hernias. Surg Endosc. 2011, in print.

8 Myers, E.; Browne, KM.; Kavanagh, DO.; Hurley M. Laparoscopic (TEP) versus Lichtenstein inguinal hernia repair: a comparison of quality-of-life outcomes. World J Surg. 2010, 34(12), 3059-64.

9. Carter, J.; Duh, QY. Laparoscopic Repair of Inguinal Hernias. World J Surg. 2011 in print.

10. Schwab, R.; Schumacher, O.; Junge, K.; et al..Biomechanical analyses of mesh fixation in TAPP and TEP hernia repair. Surg Endosc. 2008, 22(3), 731-8.

11. Taylor, C.; Layani, L.; Liew, V.; et al.. Laparoscopic inguinal hernia repair without mesh fixation, early results of a large randomised clinical trial. Surg Endosc. 2008, 22(3), 757-62.

12. Tam, KW.; Liang, HH.; Chai, CY. Outcomes of staple fixation of mesh versus nonfixation in laparoscopic total extraperitoneal inguinal repair: a meta-analysis of randomized controlled trials. World J Surg. 2010, 34(12), 3065-74.

13. McCormack, K.; Wake, BL.; Fraser, C.; et al. Transabdominal pre-peritoneal (TAPP) versus totally extraperitoneal (TEP) laparoscopic techniques for inguinal hernia repair: a systematic review. Hernia. 2005, 9(2), 109-14.

14. Swadia, ND. Laparoscopic totally extraperitoneal inguinal hernia repair: 9 year's experience. Hernia. 2011, in print.

15. Messenger, DE.; Aroori, S.; Vipond, MN. Fiveyear prospective follow-up of 430 laparoscopic totally extraperitoneal inguinal hernia repairs in 275 patients. Ann R Coll Surg Engl. 2010, 92, 201-205.

16. Myers, E.; Browne, KM.; Kavanagh, DO.; Hurley, M. Laparoscopic (TEP) versus Lichtenstein inguinal hernia repair: a comparison of quality-of-life outcomes. World J Surg. 2010, 34(12), 3059-64.

17. McCormack, K.; Wake, B.; Perez, J.; et al. Laparoscopic surgery for inguinal hernia repair: systematic review of effectiveness and economic evaluation. Health Technol Assess. 2005, 9(14), 1-203.

18. de Jonge, P.; Lloyd, A.; Horsfall, L.; et al. The measurement of chronic pain and health-related quality of life following inguinal hernia repair: a review of the literature. Hernia. 2008, 12, 561-569.

19. Srsen, D.; Druzijanic, N.; Pogorolic, Z.; et al. Quality of life analysis after open and laparoscopic inguinal hernia repair retrospective study. Hepatogastroenterology. 2008, 88, 2112-2115.

20. Bocchi, P. Shouldice's operation: Can results in a general surgical unit be the same as those in a highly specialized surgical unit? J Chir. 1993, 130, 275-7.

21. Shouldice, EB. The Shouldice repair for groin hernias. Surg Clin North Am. 2003, 83(5), 1163-87.

22. Chan, CK.; Chan, G. The Shouldice technique for the treatment of inguinal hernia. J Minim Access Surg. 2006, 2(3), 124-8.

23. Simons, MP.; Aufenacker, T.; Bay-Nielsen, M.; et al. European Hernia Society guidelines on the treatment of inguinal hernia in adult patients. Hernia. 2009, 13(4), 343-403.

24. Novik, B.; Nordin, P.; Skullman, S.; et al. More recurrences after hernia mesh fixation with shortterm absorbable sutures: A registry study of 82015 Lichtenstein repairs. Arch Surg. 2011,146(1),12-7. 
26. Everhart, JE.; Ruhl, CE. Burden of digestive diseases in the United States part I: overall and upper gastrointestinal diseases. Gastroenterology. 2009, 136, 376-386.

27. Everhart, JE.; Ruhl, CE. Burden of digestive diseases in the United States part II: lower gastrointestinal diseases. Gastroenterology. 2009, $136,741-75$.

28. Michalský, R.; Pafko, P.; Satinský, I.Operační léčení tríselné kýly. Grada, Praha, 2000.

29. Lichtenstein, IL.; Shulman, AG. Ambulatory outpatient hernia surgery. Including a new concept, introducing tension-free repair. Int Surg. 1986, 71,1-4.

30. Ger, R . The management of certain abdominal herniae by intra-abdominal closure of the neck of the sac. Preliminary communication. Ann R Coll Surg Engl. 1982, 64, 342-344.

31. Dedemadi, G.; Sgourakis, G.; Radtke, A et al. Laparoscopic versus open mesh repair for recurrent inguinal hernia: a meta-analysis of outcomes. Am J Surg. 2010, 200(2), 291-7.

32. Eklund, A.; Rudberg, C.; Leijonmarck, CE.; et al. Recurrent inguinal hernia: randomized multicenter trial comparing laparoscopic and Lichtenstein repair. Surg Endosc. 2007, 21,634-640.
33. Feliu, X.; Jaurrieta, E.; Vinas, X.; et al . Recurrent inguinal hernia: a ten-year review. $J$ Laparoendosc Adv Surg Tech A. 2004, 14,362-367.

34. Kouhia, ST.; Huttunen, R.; Silvasti, SO.; et al . Lichtenstein hernioplasty versus totally extraperitoneal laparoscopic hernioplasty in treatment of recurrent inguinal hernia-a prospective randomized trial. Ann Surg. 2009, 249, 384-387.

35. Itani, KM.; Fitzgibbons, R Jr.; Awad, SS.; et al. Management of recurrent inguinal hernias. $J \mathrm{Am}$ Coll Surg. 2009, 209(5), 653-658.

36. Wauschkuhn, C.; Schwarz, J.; Boekeler, U.; et al Laparoscopic inguinal hernia repair: gold standard in bilateral hernia repair? Results of more than 2800 patients in comparison to the literature. Surg Endosc. 2010, 24(12), 3026-3030.

37. Feliu, X.; Claveria, R.; Besora, P. Bilateral inguinal hernia repair: laparoscopic or open approach? Hernia. 2011, 15(1),15-18. 